On the Coupling of Electromagnetic Radiation into Cavities

Thomas J. T. Kwan 


\title{
ON THE COUPLING OF ELECTROMAGNETIC RADIATION INTO CAVITIES
}

\author{
by
}

\author{
Thomas J. T. Kwan
}

\begin{abstract}
A two-dimensional fully electromagnetic finitedifference time-domain code was used to investigate the penetration of electromagnetic radiation into cavities with apertures. The study used two different temporal waveforms to compare the coupling efficiency. We found that cavity modes were excited due to the induced surface current caused by the incident radiation pulse. The fast-rise pulse, which contained higher frequency components, was found to have higher coupling efficiency. The effects of other parameters such as aperture size and amplitude of the waveform were also studied.
\end{abstract}




\section{INTRODUCTION}

Behaviors of a physical system can be strongly influenced by its surrounding electromagnetic fields. In order to predict the behavior of a system in an electromagnetic environment, one has to determine the spatial and temporal profiles of the electromagnetic fields at the location of the object. Theoretical techniques for calculating the scattering of electromagnetic radiation by objects can be used in simple geometries. Furthermore, penetration of electromagnetic fields into a surrounded region through apertures has been extensively studied since the work of Kirchhoff on diffraction. Applications of Babinet's principle to electromagnetic scattining problems also yield useful results in simple geometries where geometric optics approximations are valid.

For $\mathrm{s}^{\prime} / \mathrm{stems}$ with complicated geometric configuration, the theoretical approach be zomes less rewarding and often intractable. Consequently, computer modeling of electromagnetic phenomena takes on an increasingly important role. In this work, we have used a two-dimensional fully electromagnetic code to simulate an electromagnetic pulse incident on cavities with openings. The full set of Maxwell's equations is solved in time and space (two spatial variables). Particular emphasis is placed on the correlation between the temporal profile of the electromagnetic pulse and the efficiency of coupling of electromagnetic energy into the cavity. The coupling mechanisms can be conveniently grouped into two categories, namely, diffraction through apertures and cavity mode excitation.

We have found from the computer simulation that electromagnetic fields resulting from diffraction through the aperture are the dominant components in the nearby region. The fundamental cavity mode corresponding to the dimensions of the region was also excited to an amplitude such that it was clearly observed as a superposition on the diffracted fields. More importantly, we found that the rise time of the incident electromagnetic pulse was not a strong factor in determining the 
amplitude of the electromagnetic fields in the region. However, in regions away from the aperture, the electromagnetic fields are mainly due to the excitation of cavity modes, and its amplitude was sensitive to the rise time of the incident pulse.

The organization of this paper is as follows. First, we will briefly describe the numerical methods used in our simulations. A description of the cavity geometries will be given, and the characteristics of the incident electromagnetic pulse will be specified. Next, results from our simulations will be presented. Comparisons between different geometries and incident pulses will be made. Electromagnetic fields resulting from cavity mode excitation and diffraction through apertures will be shown. Finally, we will conclude with a comparison between results obtained from slow-rise and fast-rise waveforms.

\section{NUMERICAL SIMULATION AND PROBLEM SETUP}

The computer simulation code used in this investigation is a two-dimensional electromagnetic particle-in-cell code MERLIN. The code computes the electromagnetic fields by finite differencing Maxwell's equations in space and time. The fields are self-consistently coupled to the dynamics of any charged particles present in the system through the equation of motion and the source terms in Maxwell's equations, i.e., current and charge densities. A capability of launching an electromagnetic pulse with a prescribed temporal profile was implemented in the code for this study. No charged particles were present in our simulations because the electromagnetic field amplitude under consideration would not be large enough to cause particle emission. The electromagnetic fields are advanced in time and calculated on a spatial grid throughout the systen at every time step. The time step is always chosen such that $\omega_{\max } \Delta t<1$, where $\omega_{\max }$ is the largest angular frequency of interest to retain the necessary temporal resolution for accurate calculations. The spatial constraint is the usual one that $\mathrm{k}_{\max } \Delta \mathrm{x}<1$, where $\mathrm{k}_{\max }$ is the largest wave number of interest to maintain adequate spatial resolution for the shortest wavelength in the system. 
The waveforms used in our simulations have the following analytic representation,

$$
E(t)=E_{0}(1+a / b)\left\{\exp \left[a\left(t-t_{0}\right)\right] /\left[1+a / b\left[\exp \left[(a+b)\left(t-t_{0}\right)\right]\right]\right\}\right.
$$

where $E_{o}$ is the peak amplitude of the pulse and the parameters $a$ and $b$ are chosen to correspond to the rise and fall characteristics of the pulse. The onset of the rise is denoted by $t_{0}$. The numerical evaluations for two sets of parameters are shown in Figs. $1 \mathrm{a}$ and $1 \mathrm{~b}$. Figure 1a shows the electric field has a rise time of $10 \mathrm{~ns}$, and it is the slow-rise pulse in our study. The fast-rise pulse is shown in Fig. $1 \mathrm{~b}$ where the electric field rises to its peak value of $60 \mathrm{kV} / \mathrm{m}$ in about $1 \mathrm{~ns}$. In comparison, the fastrise waveform has significantly more energy in high-frequency components (up to one gigahertz). We used these two waveforms in our study of electromagnetic coupling phenomena into cavities with openings. The walls of the cavities used in our study are treated as perfect conductors and, therefore, ohmic loss of electromagnetic fields on the walls is neglected. The electromagnetic fields inside the cavities are results of diffraction through apertures and surface current generated inside the cavities.

\section{SIMULATION RESULTS}

The simulations were performed with the two-dimensional electromagnetic relativistic particle-in-cell code, MERLIN. Since the strength of the electromagnetic fields is not large enough to cause electron emission from surfaces, the particle feature of the code was bypassed. Instead, the electromagnetic pulses in Fig. 1 were incorporated in the code. Maxwell's equations are finite-differenced in space and time while the equation of continuity is strictly observed to ensure the consistency of 

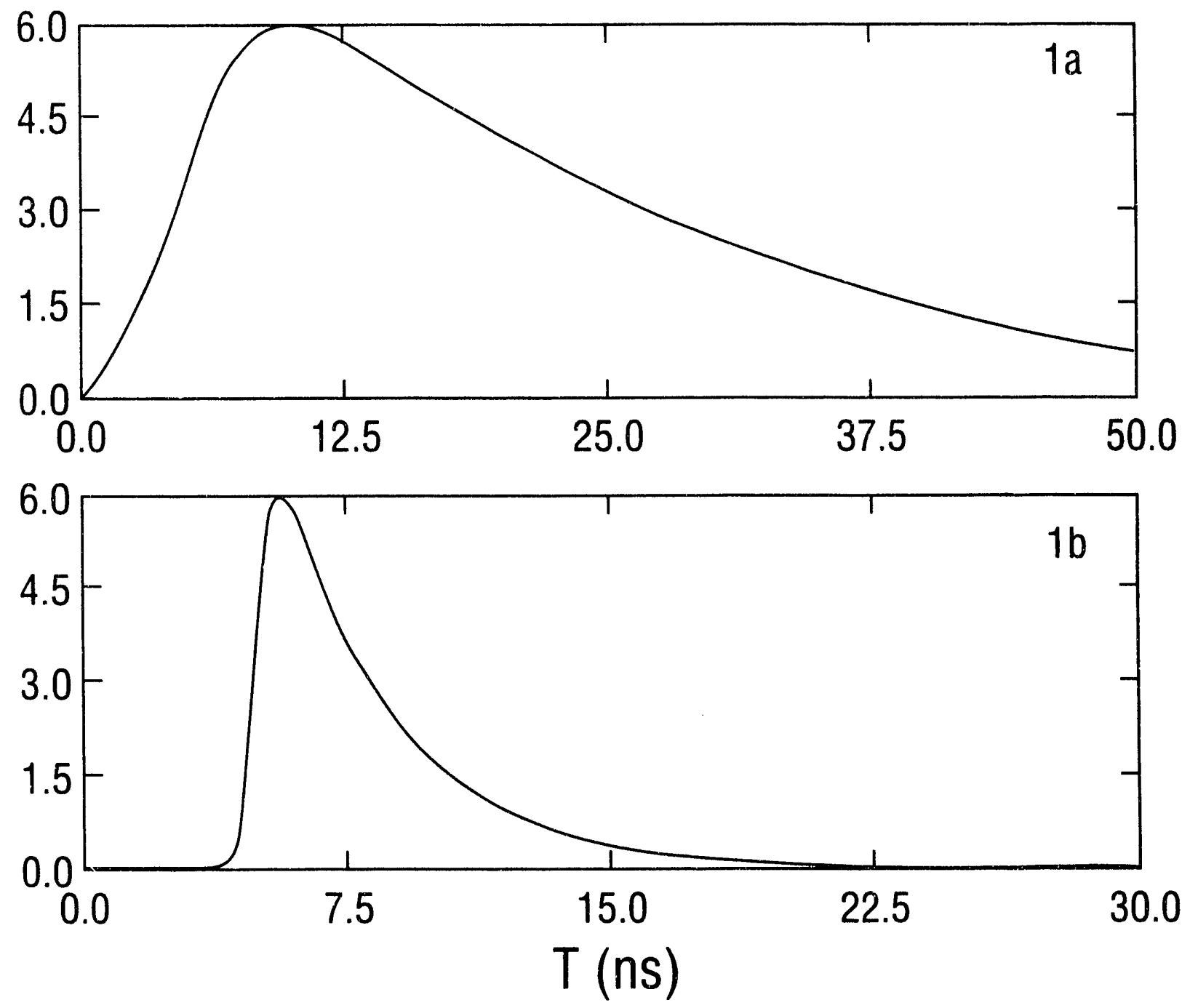

Figures $1 \mathrm{a}$ and $1 \mathrm{~b}$ : Temporal profiles of the incident electromagnetic pulses. 
the full set of Maxwell's equations. The electromagnetic fields are monitored in space and time throughout our simulations. In the following, I will describe the results from the simulation of the fast-rise electromagnetic pulse incident on a cylindrical cavity. Similar simulations were carried out with a slow-rise electromagnetic pulse, and a comparison will be presented in a tabulated table.

In Fig. 2, a cylindrical cavity with two radii of $2.39 \mathrm{~cm}$ and $7.80 \mathrm{~cm}$ is shown to have an small annular slit of $2 \mathrm{~mm}$. The radially polarized electromagnetic pulse is launched from the left boundary, and its direction of propagation is along the z-axis. The electromagnetic pulse propagates along the short section of the coaxial waveguide until it impinges on the metallic surface with a well-defined annular slit. The electromagnetic pulse undergoes diffraction at the slit into the immediate space inside the cavity: Surface current is being induced to satisfy the boundary conditions of the electromagnetic fields. The distribution of the surface current in the interior wall of the cavity leads to excitation of electromagnetic modes. Figure 3 is a contour plot of the radial electric fields at an instant of time. There are two patterns which can be readily recognized. In the region near the aperture the radiation has a characteristic spatial distribution of a dipole radiator. However, in the far away region near the zaxis, the excitation of the fundamental cavity mode is quite evident. The dipole radiation pattern near the aperture can be qualitatively explained with the aid of the Babinet principle. Here the complementary screen is an annular ring in which a radial oscillating current is induced by the incoming electromagnetic pulse. This radial current is the source for the generation of the dipole radiation observed in the simulation.

The time history of the electromagnetic fields was also monitored in our simulations. Figure 4 shows the time history of the radial electric field component at a location in the vicinity of the opening. The Fourier transform is also shown to reveal the spectral content. The temporal behavior has the general characteristics of the incoming electromagnetic pulse. However, a well-defined oscillatory component is present in the waveform. The Fourier transform indicates that the high-frequency component is at $3.5 \mathrm{GHz}$. Examination of the electromagnetic field components 


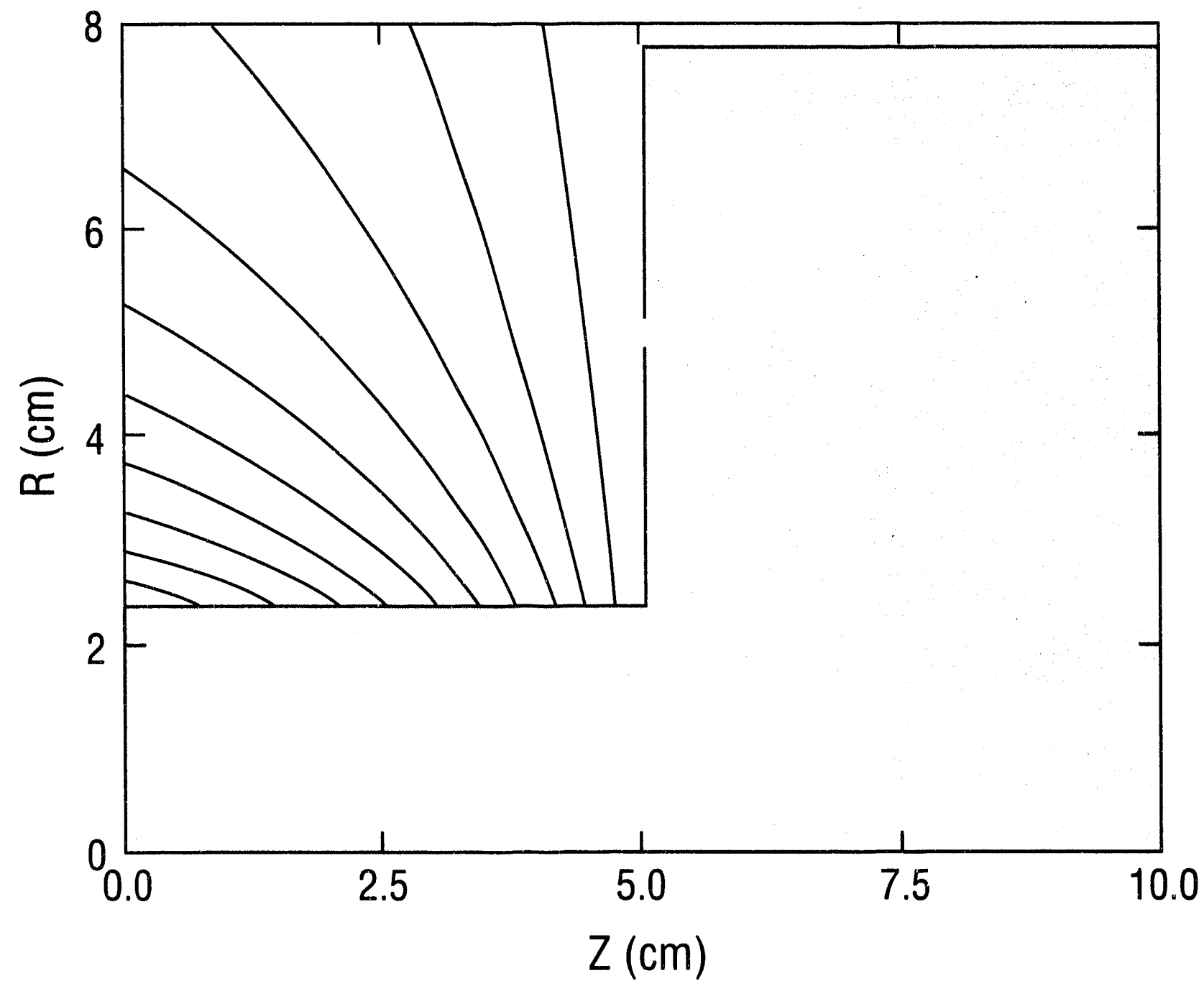

Figure 2: Constant contours of the radial electric field of the incident electromagnetic pulse in our simulation at an early time. 


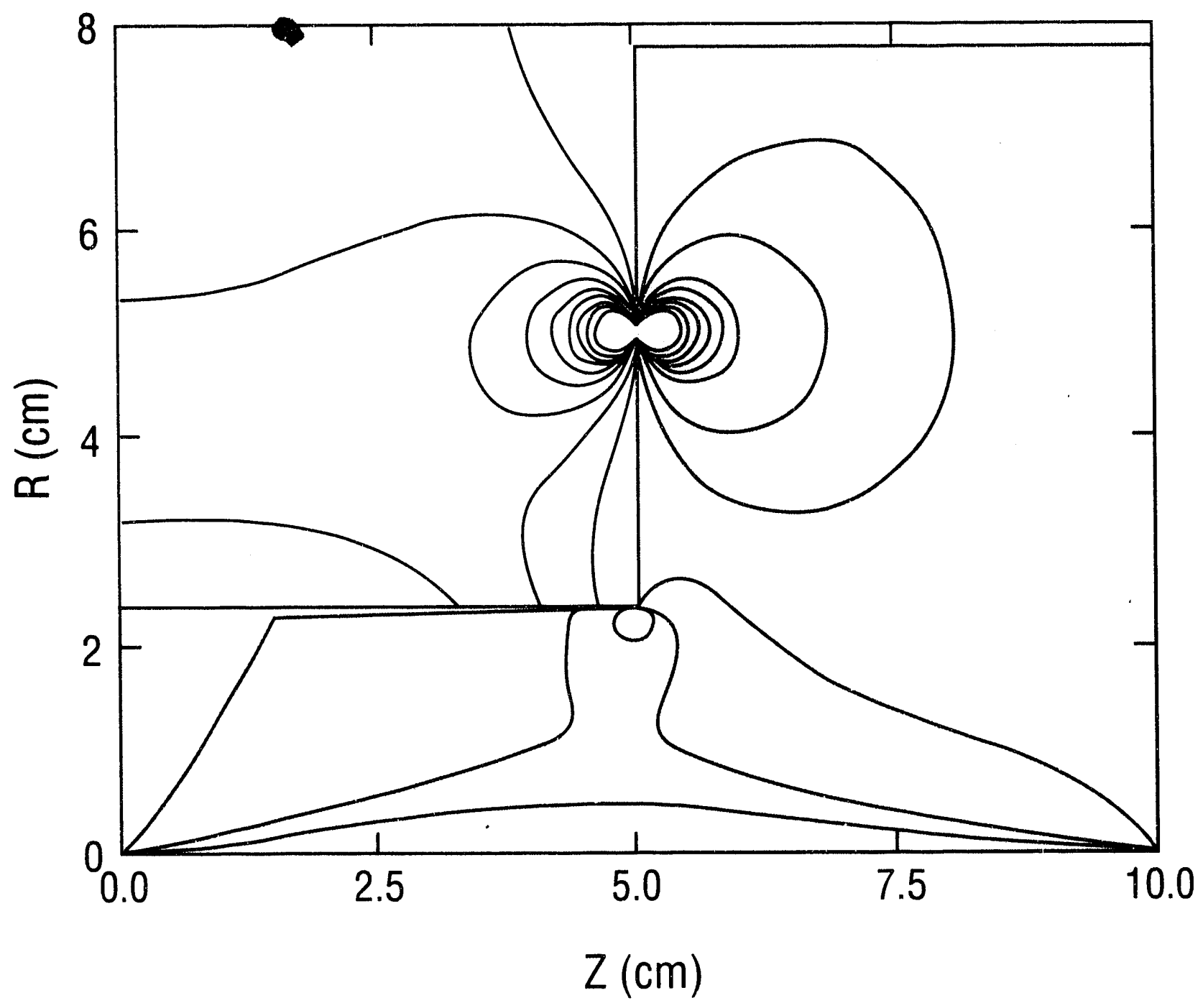

Figure 3: Constant contours of the radial electric field at a latter time shows the dipole readiation pattern and cavity mode excitation. 

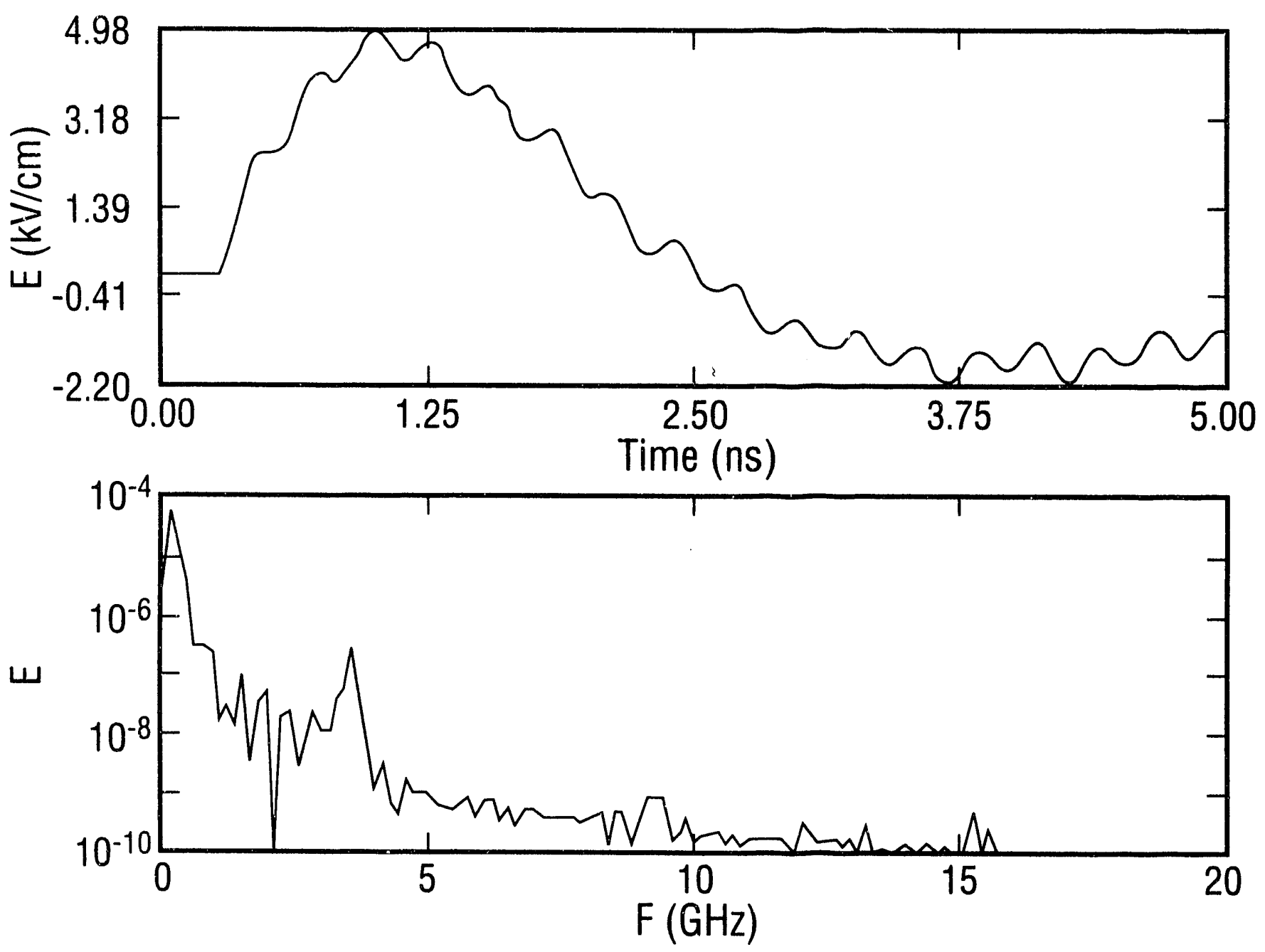

Figure 4: Time history of the radial component of electric field and its Fourier transform in the region near the aperture. 
confirms that only transverse magnetic modes existed inside the cavity. The apparently dominant mode was the $\mathrm{TM}_{011}$ which has an approximate frequency of $3.33 \mathrm{GHz}$ in good agreement with the simulation result. In this region of the cavity, the coupling efficiency can be analyzed as having two components. The part which has the similar temporal profile of the incoming pulse is a direct consequence of diffraction. On the other hand, the oscillatory component at $3.5 \mathrm{GHz}$ is due to the excitation of the $\mathrm{TM}_{011}$ mode. After obtaining the maximum amplitude of the electromagnetic field, we define the efficiency of coupling by its normalization with respect to the peak value of the incoming electromagnetic pulse. This procedure yields an efficiency of $45 \%$. The rather high efficiency, which we will show to be dependent on the aperture size primarily resulted from diffraction of the incoming pulse by the aperture.

In the lower half of the cavity away from the aperture, the dominant electromagnetic field is due to the excitation of cavity modes. In Fig. 5, we show the time history of the radial component of the electric field and its Fourier transform. It is evident that the oscillatory time behavior is the result of strong excitation of a single cavity mode. The Fourier transform of the waveform clearly indicates a dominarit frequency at $1.5 \mathrm{GHz}$. Examination of the field components in the simulation proves the cavity mode to be a transverse magnetic mode. Using the same definition, the efficiency of electromagnetic radiation coupling into this region is found to be $11 \%$.

We obtained the corresponding results for the case with a slow-rise waveform. The rise time of the electromagnetic pulse was $10 \mathrm{~ns}$. In the region adjacent to the aperture, the efficiency was found to be about $39 \%$ which is close to the result of $45 \%$ for that fast-rise pulse. Excitation of the same cavity modes was observed in the simulation, but the coupling efficiency was quite different. In the lower half of the cavity where cavity mode was the dominant component, the efficiency decreased to $1.8 \%$. In terms of energy or power couplings, the difference is even more significant because of their quadratic dependence on the electric field amplitude. 

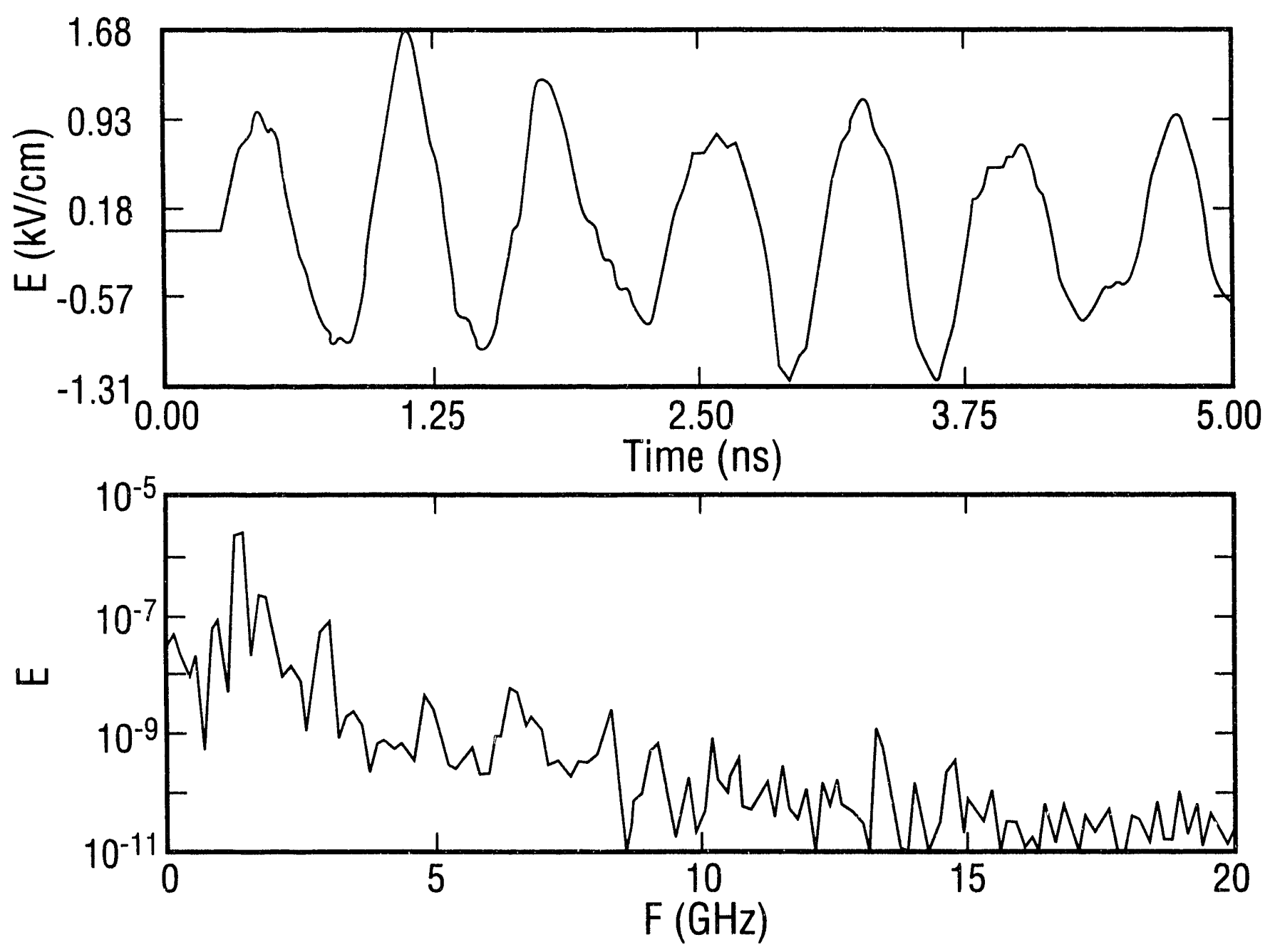

Figure 5: Time history of the radial component of electric field and its Fourier transform in the region far away from the aperture. 
The importance of the aperture size to the coupling phenomena of electromagnetic fields into the cavity was also investigated. Figure 6 shows the results from the simulations using the fast-rise pulse. The field amplitudes are normalized to the case with a $2 \mathrm{~mm}$ aperture size. Electric field strength inside the cavity increases for larger apertures, and an asymptotic behavior is clearly shown at aperture sizes greater than $6 \mathrm{~mm}$ with a $50 \%$ increase in amplitude. We also found from our simulations that the coupling efficiency is independent of the amplitude of incoming radiation pulses. Of course, this statement is only true when none of the nonlinear effects such as charged particle generation resulting from elcctrical breakdown are present in the system.
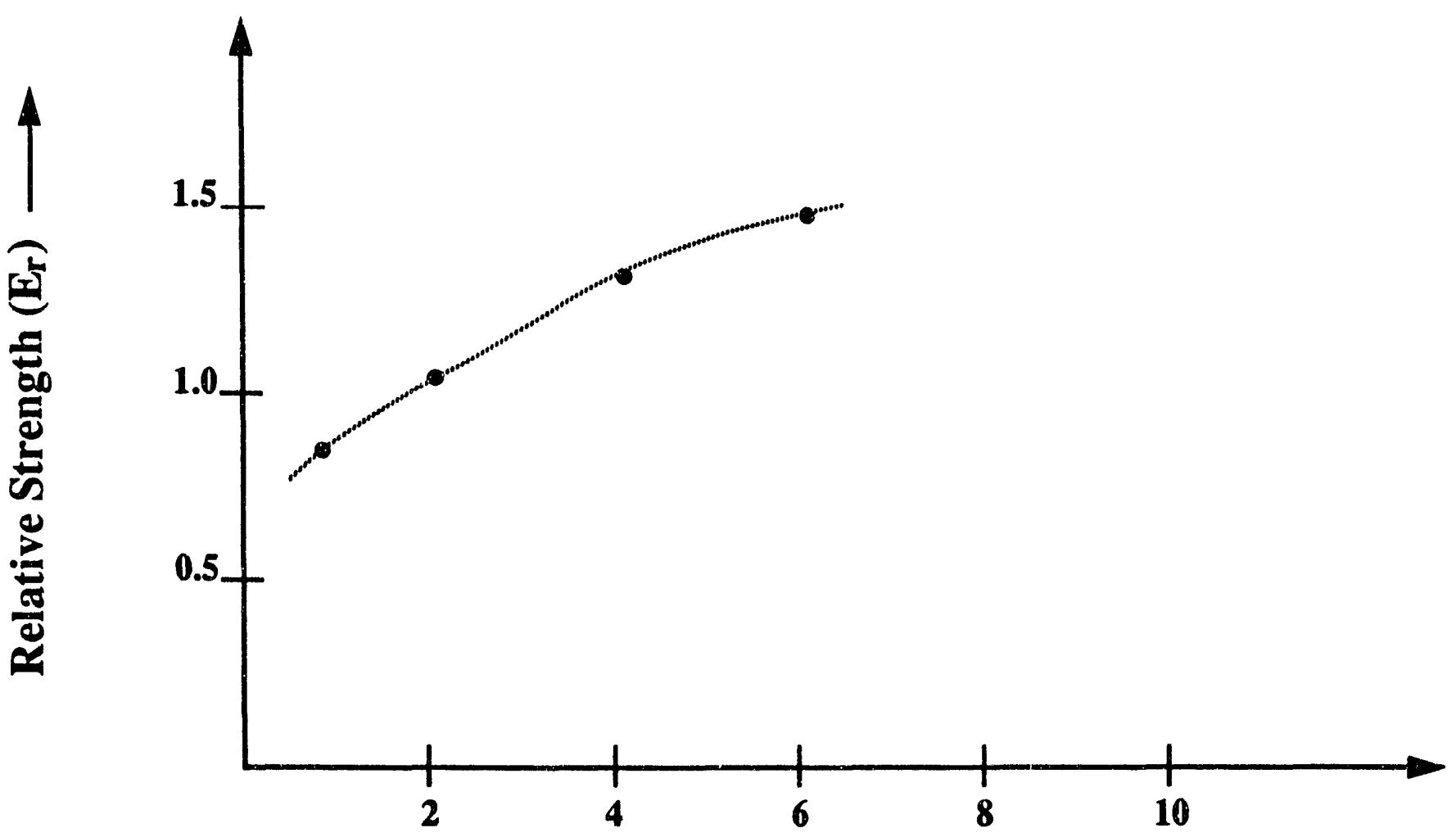

\section{Aperture Size $\Delta(\mathbf{c m})$}

Figure 6: Electric field strength inside the cavity increases and then saturates with increasing dimension of the aperture. 
Finally, we recap the results from fast-rise and slow-rise waveforms in the following table for comparison purposes.

Table I. Efficiency of electromagnetic coupling into the cavity with a $2 \mathrm{~mm}$ opening

\begin{tabular}{lcc} 
& Far Region & Nearby Region \\
\hline Fast-rise & $11 \%$ & $45 \%$ \\
\hline Slow-rise & $1.8 \%$ & $39 \%$ \\
\hline
\end{tabular}

Acknowledgments: The author would like to thank M. Bernardin, J. Brownell, K. Callahan, and B. Shafer, all of Los Alamos National Laboratory for valuable discussions. 

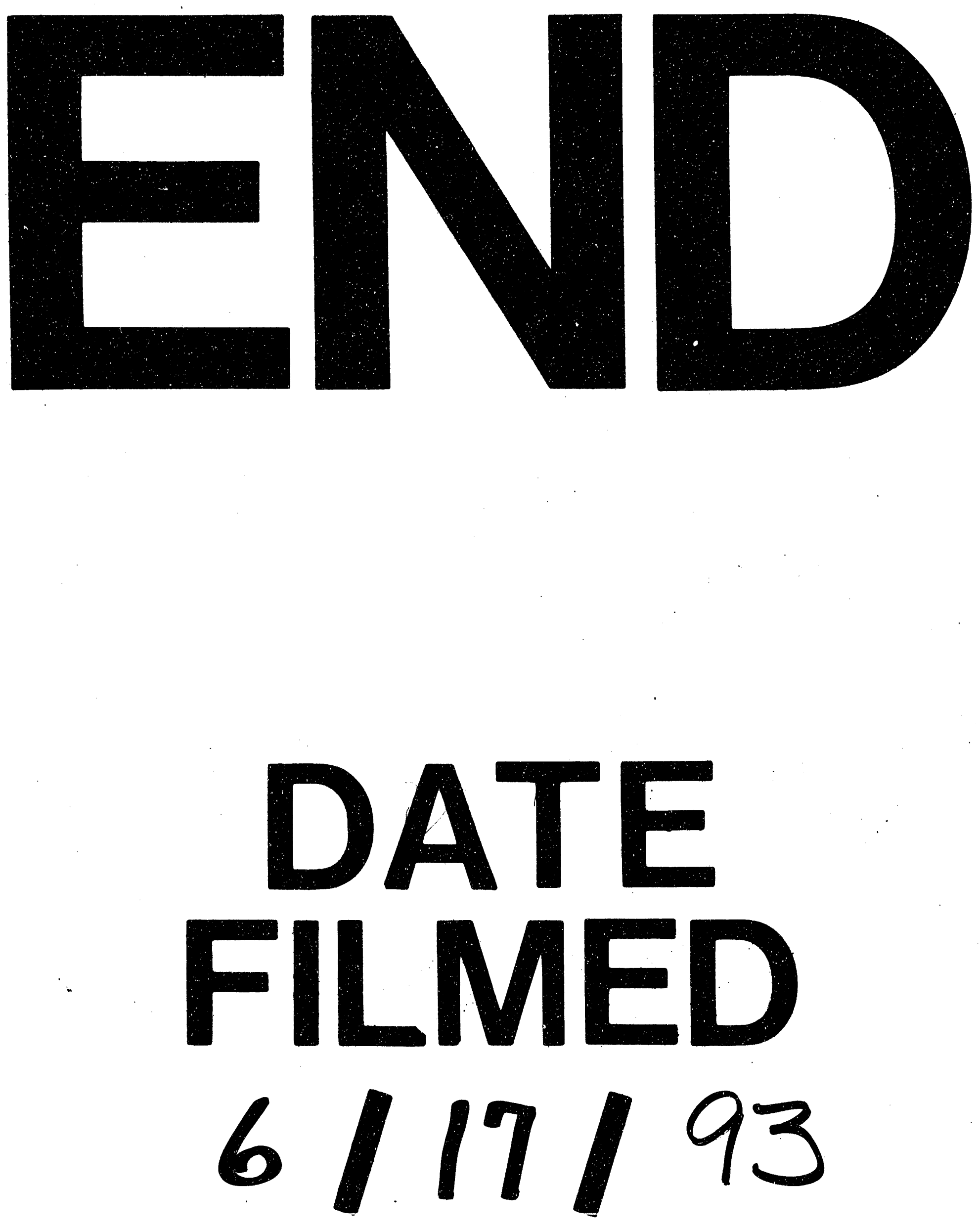
